\title{
Constitutive Expression of the Xylanase Inhibitor TAXI-III Delays Fusarium Head Blight Symptoms in Durum Wheat Transgenic Plants
}

\author{
Ilaria Moscetti, ${ }^{1}$ Silvio Tundo, ${ }^{1}$ Michela Janni, ${ }^{1}$ Luca Sella, ${ }^{2}$ Katia Gazzetti, ${ }^{2}$ Alexandra Tauzin, ${ }^{3}$ \\ Thierry Giardina, ${ }^{3}$ Stefania Masci, ${ }^{1}$ Francesco Favaron, ${ }^{2}$ and Renato D'Ovidio ${ }^{1}$ \\ ${ }^{1}$ Dipartimento di Scienze e Tecnologie per l'Agricoltura, le Foreste, la Natura e l'Energia, (DAFNE), Università della Tuscia, \\ Via S. Camillo de Lellis snc, 01100 Viterbo, Italy; ${ }^{2}$ Dipartimento TeSAF, Agripolis, Università degli studi di Padova, Viale \\ dell'Università 16, 35020 Legnaro (PD), Italy; ${ }^{3}$ ISM2/BiosCiences UMR CNRS7313, case 342, Université d'Aix-Marseille, \\ 13397 Marseille cedex 20, France
}

Submitted 29 April 2013. Accepted 2 August 2013.

Cereals contain xylanase inhibitor (XI) proteins which inhibit microbial xylanases and are considered part of the defense mechanisms to counteract microbial pathogens. Nevertheless, in planta evidence for this role has not been reported yet. Therefore, we produced a number of transgenic plants constitutively overexpressing TAXI-III, a member of the TAXI type XI that is induced by pathogen infection. Results showed that TAXI-III endows the transgenic wheat with new inhibition capacities. We also showed that TAXI-III is correctly secreted into the apoplast and possesses the expected inhibition parameters against microbial xylanases. The new inhibition properties of the transgenic plants correlate with a significant delay of Fusarium head blight disease symptoms caused by Fusarium graminearum but do not significantly influence leaf spot symptoms caused by Bipolaris sorokiniana. We showed that this contrasting result can be due to the different capacity of TAXI-III to inhibit the xylanase activity of these two fungal pathogens. These results provide, for the first time, clear evidence in planta that XI are involved in plant defense against fungal pathogens and show the potential to manipulate TAXI-III accumulation to improve wheat resistance against F. graminearum.

During the infection process, fungal pathogens secrete several cell-wall-degrading enzymes (CWDE) to breach the cell wall barrier and colonize the host tissue (Cantu et al. 2008). Among CWDE, endo- $\beta$-1,4-xylanases (xylanases; EC 3.2.1.8) are key enzymes in the degradation of xylans, a main component of cell walls of commelinoid monocot plants. According to the sequence-based glycoside hydrolase $(\mathrm{GH})$ classification (CAZy classification), xylanases are mainly grouped into families 10 (GH10) and 11 (GH11) but they have been also found in $\mathrm{GH}$ families 5, 8, and 43. The primary role of these enzymes in pathogenesis has been demonstrated for the necro-

Current address for M. Janni: IGV-CNR Via Amendola 165/A, 70126 Bari, Italy.

Corresponding author: Renato D’Ovidio; E-mail: dovidio@unitus.it

* The $\boldsymbol{e}$-Xtra logo stands for "electronic extra" and indicates that four supplementary figures and one supplementary table are published online.

(C) 2013 The American Phytopathological Society trophic fungal pathogen Botrytis cinerea because the Xyn $11 \mathrm{~A}$ gene was necessary for complete virulence during the infection of tomato leaves and grape berries (Brito et al. 2006).

The activity of microbial xylanases is controlled in vitro by specific protein inhibitors (xylanase inhibitors [XI]) localized in the plant cell wall (Dornez et al. 2010b). In wheat, three structurally different classes of proteinaceous XI have been identified: Triticum aestivum XI (TAXI) (Debyser et al. 1999), XI protein (XIP) (McLauchlan et al. 1999), and thaumatin-like XI (TLXI) (Fierens et al. 2007).

Genes encoding each class of XI have been identified. In bread wheat, three Xip genes (Xip-I, Xip-II, and Xip-III) (Elliott et al. 2002; Igawa et al. 2005; McLauchlan et al. 1999), six Taxi genes (Taxi-I, Taxi-II, Taxi-III, Taxi-IV, Taxi-IIA, and Taxi-IIB) (Igawa et al. 2004; Raedschelders et al. 2005), and one Tlxi (Fierens et al. 2007) have been characterized. In durum wheat, only Xip-II has been characterized (Elliott et al. 2009). Although there is variation in the expression of these genes, most of them are mainly expressed in the endosperm tissue where the corresponding proteins accumulate at a remarkable level (Croes et al. 2009; Dornez et al. 2010a). Immunoblot analyses also showed the lack of accumulation of XI in leaf, stem, and root tissues of wheat plants at the ripening stage (Croes et al. 2009).

A possible role of XI in plant defense is suggested by the observation that these proteins inhibit the activity of microbial xylanases in vitro and are ineffective against endogenous xylanases (Dornez et al. 2010b). The inhibition of XI against microbial xylanases is characterized by high recognition specificity between the different classes of inhibitors and xylanases. Indeed, TAXI-type and TLXI-type inhibitors inhibit GH11 but not GH10 xylanases (Fierens et al. 2007; Gebruers et al. 2004). Differently, XIP-type inhibitors are able to inhibit the xylanases of GH10 and GH11 families, although there is variability in the inhibition efficacy against xylanases of GH11 family. For example, XIP-I is not able to inhibit the GH11 xylanases (xylA and xylB) produced by Fusarium graminearum, the major causal agent of Fusarium head blight (FHB) in wheat (Beliën et al. 2005). This functional diversification of XI probably reflects the variability of xylanases secreted by the pathogens during the infection process.

Additional features that indicates XI possibly contributing to host defense include the findings that XI share sequence and structure homology with some pathogenesis-related (PR) proteins, are localized in the apoplast, and their expression is induced during stress conditions. For example, some members 
of Taxi and Xip gene families are significantly induced by wounding and treatment with jasmonic acid and methyl jasmonate (Igawa et al. 2005) and following infection with Erysiphe graminis and F. graminearum (Igawa et al. 2004, 2005, Takahashi-Ando et al. 2007).

Despite all these observations, no evidence in planta on the actual contribution of XI in plant defense has been reported thus far. Therefore, the aim of the present work was to substantiate in planta the protective role of XI during fungal pathogen infection. To this end, we produced wheat transgenic plants expressing the Taxi-III gene, an XI member that has been shown to be induced by $F$. graminearum infection (Igawa et al. 2004), and demonstrated that the transgenic plants constitutively expressing this gene showed a delay of FHB symptoms. We also showed that these plants do not show any delay in symptom development against the fungal pathogen Bipolaris sorokiniana, the causal agent of leaf spot in wheat, whose xylanase activity is not inhibited by TAXI-III.

\section{RESULTS}

\section{Taxi-III isolation and expression}

following $\boldsymbol{F}$. graminearum infection in durum wheat.

In order to select the Taxi-III gene to be used in the genetic transformation of durum wheat 'Svevo', we cloned Taxi-III from this cultivar and from 'Chinese Spring' and 'Bobwhite' bread wheat by using the primer pair $101 \mathrm{sp}$ (For)/101sp(Rev) developed from Taxi-IIII of T. aestivum 'Norin61' (accession number AB114627) (Igawa et al. 2004). The coding region of all three genes is $1,206 \mathrm{bp}$ and the deduced proteins contain a signal peptide of 21 residues (predicted by the SignalP program) for targeting to the apoplast. Each mature protein is composed of 380 amino acids with a molecular mass of approximately 39,000. Sequence comparison between these genes (Supplementary Fig. S1) showed that Taxi-III from Svevo is identical to that of Norin61 and differs by two synonymous substitutions from those of Bobwhite and Chinese Spring. Based on these results, we used this latter gene to prepare the construct for particle bombardment of durum wheat Svevo in order to facilitate the distinction between the transgenic and the endogenous Taxi-III.

Because Taxi-III has been reported to be induced in bread wheat following $F$. graminearum infection, we verified whether this induction also occurs in Svevo durum wheat. Quantitative reverse-transcriptase polymerase chain reaction (qRT-PCR) analysis with primers Taxi-III170F/Taxi-III5R showed that Taxi-III underwent a significant increase at $24 \mathrm{~h}$ postinfection (hpi), with approximately 40-fold increase (42.28 \pm 10.96$)$ compared with the corresponding mock-inoculated sample. Similarly, qRT-PCR with a primer pair specific for the homologous genes TaPr-1-1, TaPr-1-2, and TaPr-1-3 (hereafter named TaPr-1-1/2/3) (Lu et al. 2011) used as control of gene induction underwent approximately 10 -fold $(10.51 \pm 2.72)$ increase compared with the mock-inoculated control sample.

\section{Production of wheat transgenic plants expressing TAXI-IIICS.}

In total, 1,932 T. durum Svevo immature embryos were cotransformed using pUbi::Taxi-IIICS and pUbi::bar in a single bombardment experiment. Taxi-IIICS was prepared under control of the constitutive maize ubiquitin (Ubi-1) promoter in order to accumulate the corresponding protein also in tissues that normally do not accumulate TAXI-III or other XI, as leaves and flowering spikes. This strategy was used to facilitate the identification of TAXI-IIICS activity and the possible correlation between TAXI-IIICS accumulation and disease symptom development.
In all, 71 independent regenerated plants were obtained and analyzed for the presence of the transgene by PCR using the specific primers Ubi-49F/TaxiIII2R. Twenty-two $\mathrm{T}_{0}$ independent transgenic plants resistant to the bialaphos herbicide and containing Taxi-IIICS were obtained, for a transformation efficiency of $1.3 \%$ that is within the typical efficiency obtained in stable wheat transformation.

Inhibition activity of transgenic plants containing TaxiIIICS, hereafter called TAXI-III plants, was analyzed on an agarose diffusion assay using total leaf protein extract against the Aspergillus niger M4 xylanase (AnM4Xyl). The inhibition assays showed that total protein extract $(20 \mu \mathrm{g})$ of 9 of $22 \mathrm{~T}_{1}$ TAXI-III plants inhibited to a varying extent (30-100\%) the activity of AnM4Xyl (Table 1). In contrast, total protein extract from the control plants did not affect the AnM4Xyl activity (Table 1). Moreover, when protein extract of TAXI-III plants was boiled no inhibition activity was observed, excluding the possibility of a non proteinaceous inhibition.

Three TAXI-III lines, MJ30-6, MJ30-10 and MJ30-23 showing a high inhibition activity against AnM4Xyl were selected for subsequent analyses. $T_{1}$ plants of these lines did not show any significant difference in morphology and growth compared with untransformed plants ( $T$. durum Svevo). $\mathrm{T}_{2}$ and $\mathrm{T}_{3}$ progenies from all three primary transformants maintained the inhibition activity against AnM4Xyl, although random transgene silencing occurred. This phenomenon has been observed since the $T_{1}$ generation and increased in the subsequent $T_{2}$ and $\mathrm{T}_{3}$ generations. Because transgene silencing occurred randomly in the progenies and also during plant growth and development, all the transgenic plants were monitored during growth and only those showing inhibition activity against AnM4Xyl until flowering were used in subsequent experiments.

\section{Molecular characterization, inhibition activity, and apoplastic accumulation of TAXI-IIICS in selected transgenic durum wheat lines.}

Genomic DNA blots of the selected lines (MJ30-6, MJ3010, and MJ30-23) were performed using the entire coding region of Taxi-IIICS as probe. Digestion with BamHI restriction enzyme, which causes the excision of the Taxi-IIICS coding region from the pUbi::Taxi-IIICS (Fig. 1A), revealed the expected hybridization signal of approximately $1,200 \mathrm{bp}$ in all transgenic lines (Fig. 1B). Digestion with SphI restriction enzyme, which cuts once within the pUbi::Taxi-IIICS, produced in all the transgenic lines a main hybridizing fragment of

Table 1. Inhibition activity of total protein extract of $T_{1}$ TAXI-III plants and wild-type (WT) plants against Aspergillus niger xylanase AnM4Xyl as determined by radial gel diffusion assay ${ }^{\mathrm{a}}$

\begin{tabular}{lc}
\hline Lines & Relative inhibition activity $(\boldsymbol{\%})$ \\
\hline WT & 0 \\
MJ30-1 & $27 \pm 4.04$ \\
MJ30-6 & $100 \pm 6.15$ \\
MJ30-10 & $100 \pm 7.23$ \\
MJ30-10b & $100 \pm 8.66$ \\
MJ30-12a & $33 \pm 6.33$ \\
MJ30-23 & $100 \pm 7.13$ \\
MJ30-36 & $100 \pm 6.21$ \\
MJ30-52 & $40 \pm 3.09$ \\
MJ30-68 & $36 \pm 7.89$ \\
\hline
\end{tabular}

a Inhibition activity of total protein extract of each transgenic line is expressed as relative percentage, considering $0 \%$ of inhibition as the halo produced by AnM4Xyl in the presence of the protein extract of the WT plants and $100 \%$ of inhibition as the absence of halo. Data represent the average \pm standard errors of three replicates by using $20 \mu \mathrm{g}$ of total protein extract and $0.005 \mathrm{U}$ of $A$. niger M4 xylanase. Boiled protein extract samples did not exhibit any inhibitory activity against $0.005 \mathrm{U}$ of $A$. niger M4 xylanase. 
approximately $5.9 \mathrm{~kb}$, corresponding to the size of the entire construct (Fig. 1C). This result suggests the integration of multiple copies of the construct in head-to-tail array into the genome of the transgenic lines. Additional hybridizing fragments were also present in all three transgenic lines. Some of these hybridization fragments were also present in the control plants and probably correspond to endogenous Taxi genes, whereas others were specific to each transgenic line and most likely represent different insertion sites or rearranged copies of pUbi::Taxi-IIICS as well as the terminal fragment of the transgene array (Fig. 1C).

RT-PCR analysis showed that Taxi-III was clearly expressed in the leaves of all three transgenic lines (MJ30-6, MJ30-10, and MJ30-23). Conversely, a weak expression of endogenous Taxi-III was observed in the control plants of Svevo. qRT-PCR showed that the level of expression of Taxi-III in spikelets was similar in all three transgenic lines and more than 500-fold higher than in control plants (Fig. 2A).

In agreement with the results at the RNA level, total protein extract from $\mathrm{T}_{1}$ plants of all three lines inhibited AnM4Xyl at a similar extent. In particular, less than $5 \mu \mathrm{g}$ of total leaf protein extract each of MJ30-6, MJ30-23, and MJ30-10 was sufficient to inhibit the AnM4Xyl activity by $50 \%$ (Fig. 2B), and $10 \mu \mathrm{g}$ inhibited this enzyme to completion (Fig. 2C). Similarly, total protein extract from flowering spikes of transgenic plants
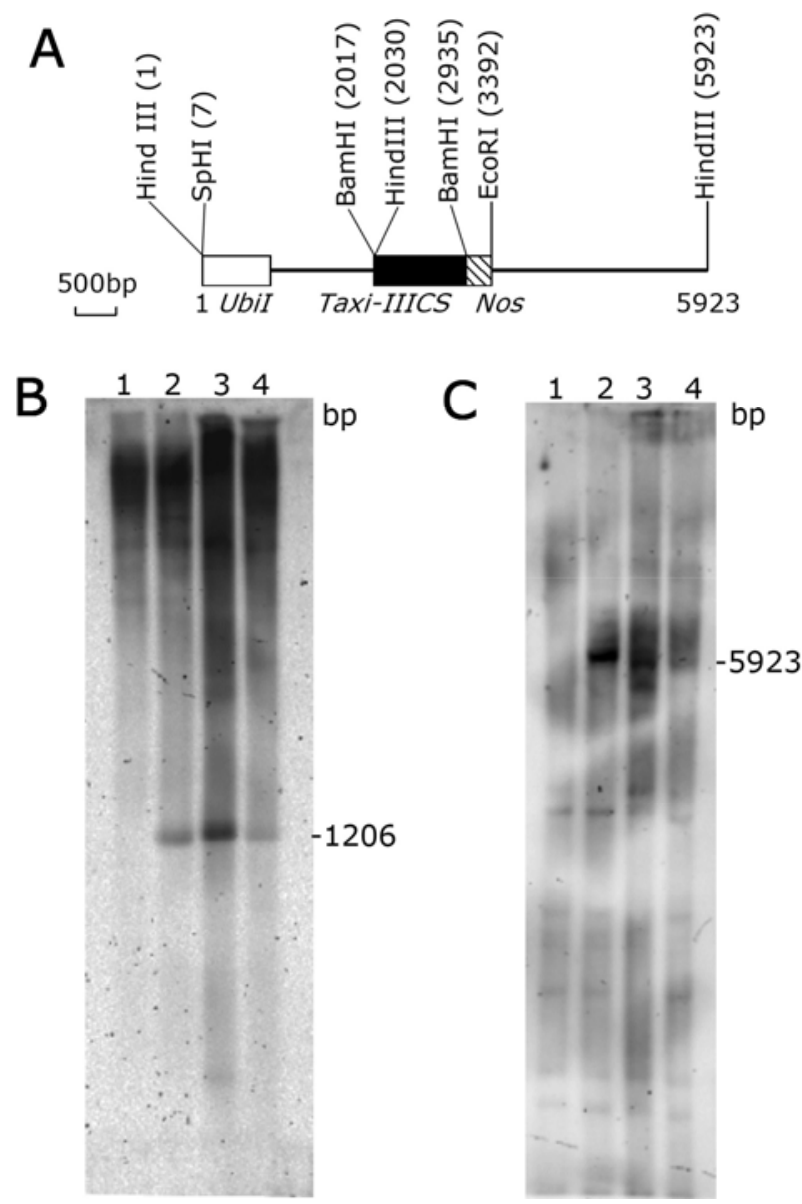

Fig. 1. Schematic representation of the pUbi::Taxi-IIICS construct and Southern blots of regenerated transgenic lines. A, The pUbi::Taxi IIICS construct was prepared by cloning the Taxi IIICS gene into the BamHI site of pAHC17 under control of the constitutive maize Ubiquitin1 promoter and NOS terminator. Genomic DNA $(10 \mu \mathrm{g})$ of $\mathrm{T}_{2}$ transgenic lines was digested with $\mathbf{B}$, Bam HI and $\mathbf{C}, S p h \mathrm{I}$ probed with a digoxigenin-labeled coding region of Taxi IIICS. Lane 1, Triticum durum 'Svevo' (untransformed control); lane 2, MJ30-6; lane 3, MJ30-10, lane 4, MJ30-23. inhibited AnM4Xyl to completion (Fig. 2C). In contrast, no inhibition activity was observed with total protein extract (up to $30 \mu \mathrm{g}$ ) from leaves or flowering spikes of control plants (Fig. 2C). In the experiments involving flowering spikes, the ovary was removed before extraction to avoid the interference
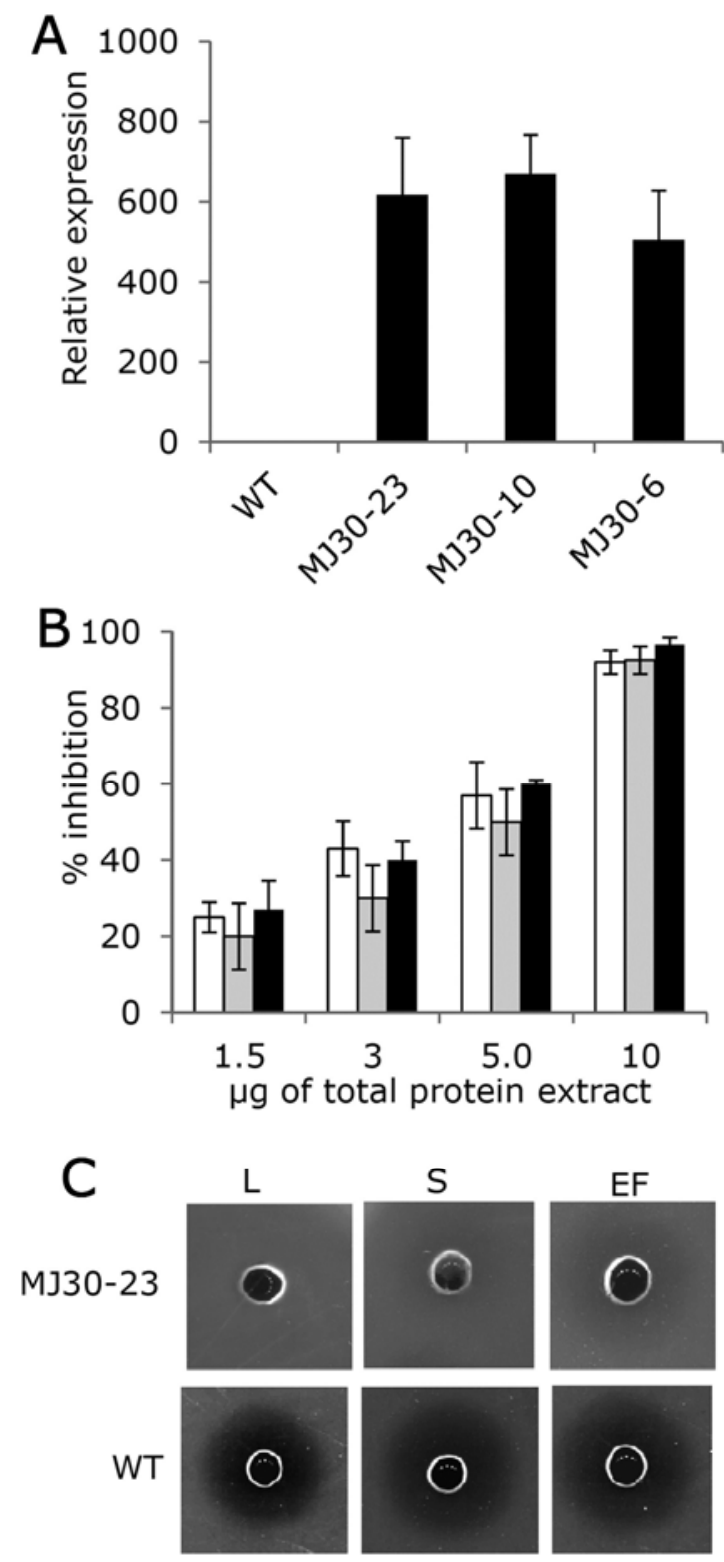

Fig. 2. Expression and xylanase inhibitor (XI) activity of selected wheat lines overexpressing Taxi IIICS. A, Quantitative reverse-transcriptase polymerase chain reaction on RNA extracted from spikelets of control plants (wild type [WT]) and three transgenic lines (MJ30-23, MJ30-10, and MJ20-6) using primers specific for Taxi-III. Quantification of gene expression was performed using the comparative $2-\Delta \Delta C T$ method. Relative expression of Taxi III is reported as fold increase of transcript level in spikelets at anthesis (Zadoks stage 68), deprived of the ovary, relative to values determined in control plants. Actin was used as housekeeping gene. Bars represent average values \pm standard errors of two biological replicates and six technical replicates. B, Agarose diffusion assays for XI activity of total leaf protein extract of transgenic line MJ30-23 (white), MJ3010 (gray), and MJ30-6 (black). Assays were performed using different amounts of total protein extract against AnM4Xyl. Bars represent the average \pm standard error of at least three replicates. C, Agarose diffusion assay for XI activity of total protein extract $(10 \mu \mathrm{g}$ ) from leaf (Zadoks stage 12) and spikelets (Zadoks stage 68), and of extracellular fluids ( $3 \mu \mathrm{g}$ ) of transgenic line MJ30-23 and WT plants (Triticum durum 'Svevo', WT) against AnM4Xyl; the absence of the halo indicates the presence of XI activity. L, leaf; S, spikelets; EF, extracellular fluids. 
with possible endogenous XI activity accumulated in the early stage of embryo development.

Because TAXI-IIICS contains a predicted signal peptide for the apoplastic targeting, we extracted and assayed the extracellular leaf fluids (EFs) of line MJ30-23 and control plants against AnM4Xyl. An agarose diffusion assay showed a clear inhibition activity in the EF of MJ30-23 and a lack of this activity in the EF of control plants (Fig. 2C), indicating that TAXI-III is targeted into the apoplastic compartment. EFs from both samples had negligible glucose-6-phosphate dehydrogenase activity, ruling out cytoplasmic contamination (data not shown).

TAXI-III purification and inhibition properties against the xylanase activity of the fungal pathogens $F$. graminearum and $B$. sorokiniana.

TAXI-IIICS was purified from total leaf protein extract of line MJ30-23 by affinity chromatography on a SepharoseAnM4Xyl column. Sodium dodecyl sulfate polyacrylamide gel electrophoresis (SDS-PAGE) showed a single band of the expected size of approximately $40 \mathrm{kDa}$ (Supplementary Fig. S2A) on those fractions containing inhibition activity against AnM4xyl. Mass spectrometry (MS) (Supplementary Fig. S3) and N-terminal sequence (Supplementary Fig. S4) confirmed the correspondence of the protein. This latter analysis also showed that the mature TAXI-IIICS starts with Lys at position 19 (KGLPVLAPVT) of the complete deduced protein from the Taxi-IIICS gene. This result is slightly different from that predicted by SignalP that indicated the putative cleavage sites between residues 21 and 22 .

Purified TAXI-IIICS was used to determine the kinetics parameters of the inhibitor against AnM4Xyl. Dinitrosalicylic acid (DNS) assays showed that this enzyme exhibits $K_{m}$ of $0.018 \mathrm{mM}$ and $k_{\text {cat }}$ of $5855.60 \mathrm{~s}^{-1}$ on soluble wheat low-viscos-

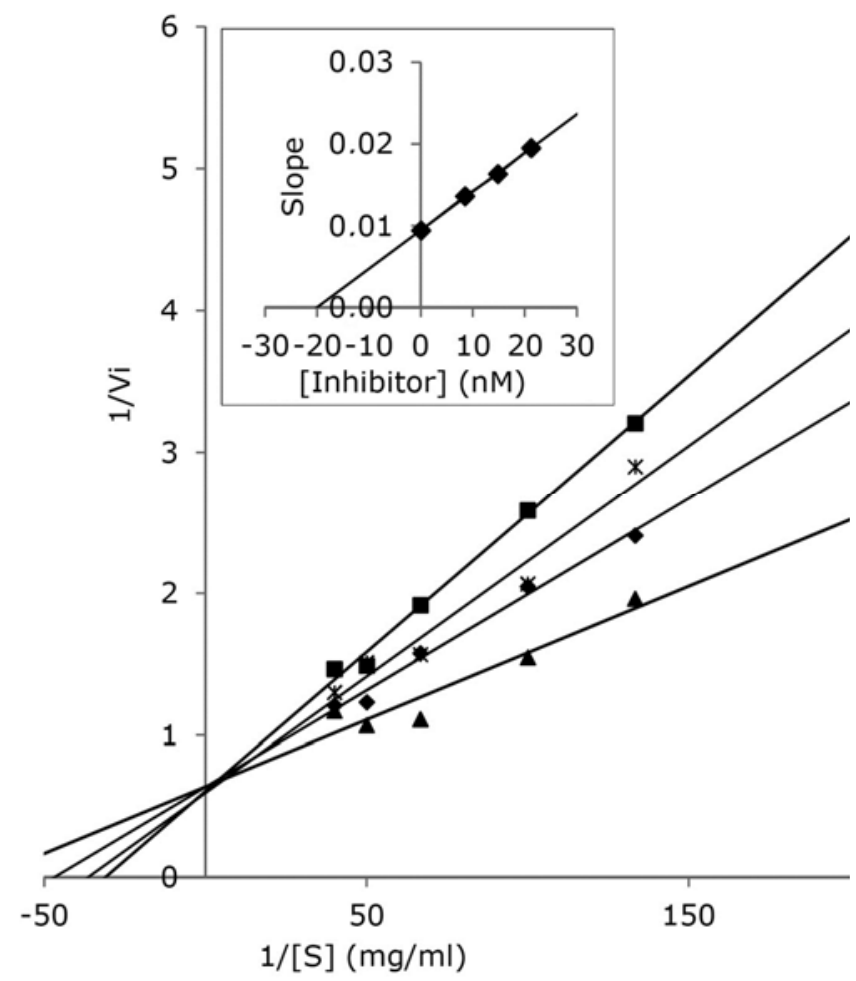

Fig. 3. Kinetic analyses of TAXI-IIICS. Double-reciprocal plots of the inhibition of AnM4Xyl by TAXI-IIICS using low-viscosity wheat arabinoxylan as substrate. Xylanase activity with no inhibitor $(\mathbf{A})$ and in the presence of $8.0 \mathrm{nM}(\diamond), 14.0 \mathrm{nM}(*)$, and $20.0 \mathrm{nM}(\boldsymbol{\square})$ inhibitor. The insert shows the secondary plot of slopes from the double-reciprocal plot against inhibitor concentration to give $K_{i}$. ity arabinoxylan. In the presence of purified TAXI-IIICS, the Vmax did not change but the $K_{m}$ increased with increasing concentrations of the purified inhibitor (Fig. 3). Line convergence at the $\mathrm{Y}$-axis on the double reciprocal plot indicated that the inhibition is competitive (Fig. 3). A secondary plot of slopes against TAXI-IIICS concentration gave an inhibition constant, $K_{i}$, of $18.8 \mathrm{nM}$ at pH 5 (Fig. 3).

Purified TAXI-IIICS was also assayed against the xylanase activity of the fungal pathogens $B$. sorokiniana and $F$. graminearum grown on wheat bran as sole carbon source. TAXIIIICS only partially inhibited the total xylanase activity of $F$. graminearum. Conversely, no inhibition activity was observed against the total xylanase activity of $B$. sorokiniana (Fig. 4A).

Inhibition assays using total protein extracts from leaves or spike showed the same results; that is, an incomplete inhibition and no inhibition against total xylanase activity of $F$. graminearum and B. sorokiniana, respectively (Fig. 4B).

In order to verify the origin of the partial inhibition of TAXIIIICS against the total activity of $F$. graminearum, we expressed in Pichia pastoris four endoxylanase genes-FGSG_11487, FGSG_10999, FGSG_11304, and FGSG_03624-whose corresponding transcripts accumulated during infection of wheat spikes (Lysøe et al. 2011; Paper et al. 2007; Sella et al. 2013). Culture filtrates of $P$. pastoris expressing these xylanases were used to perform DNS assays with purified TAXI-IIICS or total protein extracts from the transgenic line MJ30-23. The xylanase FGSG_03624 was completely inhibited by $250 \mathrm{ng}$ of TAXIIIICS, whereas the same amount of inhibitor reduced by $75 \%$
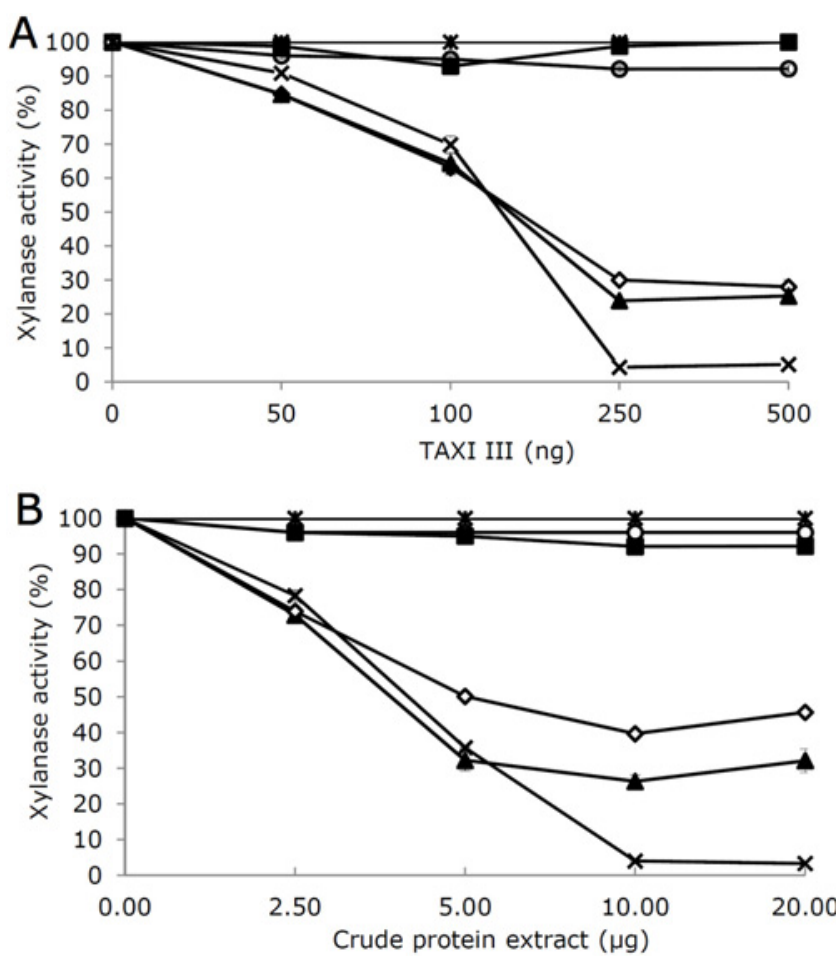

Fig. 4. Inhibition activity of purified TAXI-IIICS or crude protein extract of transgenic line MJ30-23 against Fusarium graminearum and Bipolaris sorokiniana xylanase activities. A, Xylanase activities determined in the presence of increasing amounts of purified TAXI-IIICS. B, Xylanase activities determined in the presence of increasing amounts of leaf crude protein extract of transgenic line MJ30-23 overexpressing TAXI IIICS. Inhibition assays performed against the same xylanases in the same conditions with leaf crude protein extract of control plants (Triticum durum 'Svevo') did not show any inhibition activity. Pichia pastoris culture filtrates FGSG_11487 (*), FGSG_10999 (山), FGSG_11304 (O), and FGSG_03624 (x); culture filtrates of $F$ graminearum $(\diamond)$ and B. sorokiniana $(\overline{\mathbf{\square}})$ were grown on wheat bran. 
the activity of xylanase FGSG_10999. Increased amount of the inhibitor did not modify the inhibition capacity against these two xylanases (Fig. 4A). Conversely, the activity of FGSG 11487 and FGSG_11304 was unaffected by TAXI-IIICS (Fig. 4A). Similar results were obtained by performing the inhibition assays using total protein extract of the transgenic line MJ30-23 (Fig. 4B).

\section{Transgenic wheat TAXI-III lines show increased resistance against $F$. graminearum infection but not against $B$. sorokiniana.}

Infection experiments with $B$. sorokiniana were performed on the first emerged leaf of TAXI-III lines MJ30-6, MJ30-10, and MJ30-23 and wild-type plants (T. durum Svevo). Disease symptoms were visible $48 \mathrm{hpi}$ and appeared as reddish-brown spots of variable size on the leaf surface. In order to facilitate the analysis of single lesions, data were collected 72 hpi. Disease symptoms were evaluated as the ratio between leaf area showing symptoms and the total leaf area, expressed as percentage (Fig. 5). Statistical analysis of data indicated that none of the transgenic lines showed a significant reduction of disease symptoms compared with control plants (Fig. 5).

Flowering spikes of transgenic lines MJ30-6, MJ30-10, and MJ30-23 and wild-type plants (T. durum Svevo) were also subjected to infection with the fungal pathogen $F$. graminearum. Infection experiments were performed by point inoculation of the opposite central spikelets in primary spikes (Zadoks stage 68) of transgenic and control plants. FHB disease symptoms, manifested as spikelet bleaching, usually appeared 3 days postinfection (dpi) and the spread of the disease was visually examined for a period of 20 days (Fig. 6). All three transgenic lines showed a significant reduction of symptoms from 3 to 11 dpi compared with control plants. Line MJ30-6 continued to show a significant symptom reduction until 14 dpi, whereas line MJ30-23 showed symptom reduction until the end of the analysis (20 dpi). However, the maximum symptom reduction was observed between 8 and $10 \mathrm{dpi}$, when the reduction of FHB symptoms was higher than $25 \%$ for all three transgenic lines compared with control plants.

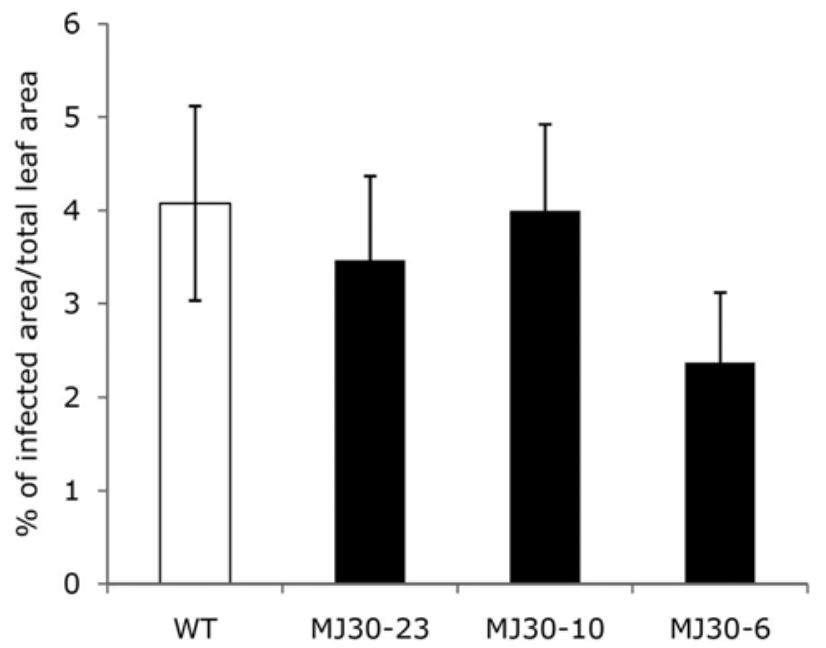

Fig. 5. Quantification of symptomatic leaf area of transgenic lines MJ30-6, MJ30-10, and MJ30-23 and wild-type (WT) plants (Triticum durum 'Svevo') inoculated with conidia of Bipolaris sorokiniana. Symptom severity (lesion expansion area [pixel]/total leaf area [pixel]) $\times 100$ at $72 \mathrm{~h}$ postinfection. Data represent the average \pm standard errors of four experiments for control plants, three experiments for lines MJ30-10 and MJ3023, and two experiments for MJ30-6, all performed with at least 13 replicates. The average values were not significantly different according to Student's $t$ test.
The reduced FHB symptoms were associated with a reduced accumulation of fungal biomass in the caryopses of transgenic plants. qPCR analyses with primers specific for the fungal TRI-6 gene, Tri6_10F and Tri6_4R (Horevaj et al. 2011), on total DNA extracted from whole flour of the transgenic lines and control plants showed approximately twofold reduced biomass in the transgenic samples $(0.47 \pm 0.036$ and $0.56 \pm 0.21$ for MJ30-23 and MJ30-6, respectively) compared with the control sample (T. durum Svevo). Probably, this reflects a slightly reduced growth of the pathogen in the early and advanced phases of the infection of the transgenic plants compared with the control.

\section{DISCUSSION}

Transgenic expression of Taxi-IIICS under control of the constitutive maize $U b i-1$ promoter endowed the transgenic durum wheat plants with the capacity to accumulate the transgene-encoded TAXI-IIICS in all tissues. This accumulation did not cause any major phenotypic change, although transgene silencing occurred at quite a high frequency in the progeny and during plant growth. The accumulation of TAXI-IIICS was easily identified by the presence of inhibition activity against microbial xylanases in total protein extracts of transgenic tissues, including those that, in the untransformed plants, do not show any XI activity.

TAXI-IIICS purified from transgenic tissue showed a competitive inhibition against AnM4Xyl, with a $K_{i}$ of $18.8 \mathrm{nM}$ at $\mathrm{pH}$ 5. This $K_{i}$ value is similar to that found for TAXI-I (20 nM) (Gebruers et al. 2004), one of the best studied and most represented XI in wheat endosperm. Differently from TAXI-I, TAXIIII has not been detected in wheat endosperm, also following infection with a $F$. graminearum strain exhibiting a reduced pathogenicity (Dornez et al. 2010a). Nevertheless, our transcript analyses showed that Taxi-IIISV is induced in the spikelets by $F$. graminearum infection, confirming previous results obtained on bread wheat (Igawa et al. 2005).

Although the induction of the endogenous Taxi-III following fungal infection can contribute to an increase of xylanase inhibition activity, the level reached is probably not sufficient to contrast effectively against the activity of the xylanases secreted by pathogens. Differently, the TAXI-III plants possess a high level of xylanase inhibition activity and this is related to a

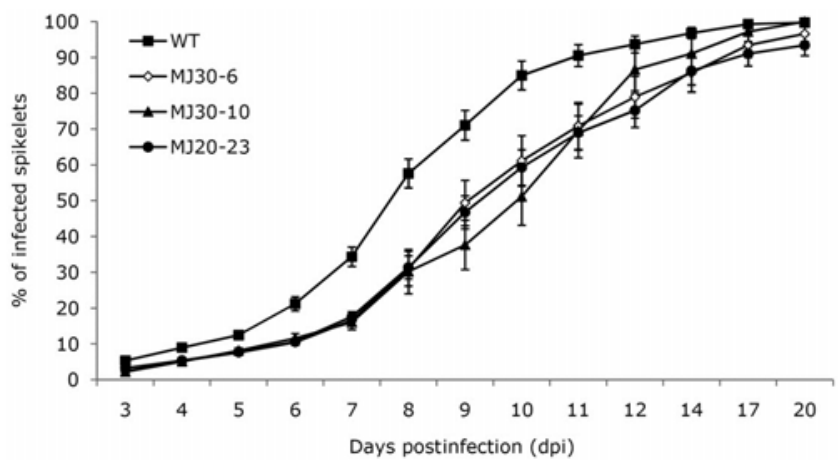

Fig. 6. Time-course analysis of Fusarium head blight symptom development following Fusarium graminearum infection of transgenic lines MJ30-6, MJ30-10, and MJ30-23 and wild-type (WT) plants (Triticum durum 'Svevo'). Disease symptoms are expressed as percentage of spikelets showing symptoms on the total number of spikelets per spike. Data represent the average \pm standard errors of three experiments for line MJ30-23 and WT plants, two experiments for line MJ30-6 and line MJ30-10, performed with at least 12 plants per genotype. Average values of lines MJ3023, MJ30-10, and MJ30-6 are significantly different from the WT plants according to Student's $t$ test $(P \leq 0.01$ or 0.05$)$ until 20,14 , and 11 days postinfection (dpi), respectively. 
delay of disease symptom development. Indeed, the TAXI-III plants are characterized by the development of symptoms from the inoculated spikelets but showed reduced spread of symptoms to the surrounding spikelets compared with control plants. The reduction of FHB symptoms is particularly evident in the early phase of fungal infection ( 8 to $10 \mathrm{dpi}$ ), when the infection level is reduced by approximately $25 \%$. Probably, this outcome is due to the reduced ability of $F$. graminearum to degrade the cell wall xylans of transgenic plants. These polysaccharides are major components of the wheat cell wall and, for their degradation, $F$. graminearum secretes a number of xylanases. Among them, six different endoxylanase genes are clearly expressed during wheat infection (Lysøe et al. 2011; Paper et al. 2007; Sella et al. 2013). These genes code for xylanases with different catalytic properties. For example, those xylanases belonging to family GH11 are active on unsubstituted regions of xylan, whereas those of family GH10 can also hydrolyze decorated forms of arabinoxylans (Biely et al. 1997). The contemporary secretion of xylanases with different catalytic properties probably reflects the heterogeneity of cell wall xylans and the complexity of the cell wall.

To contrast this variability of xylanases, plants have evolved different types of XI with different inhibition properties (Dornez et al. 2010b). Here, we demonstrated that TAXI-III inhibits the F. graminearum xylanases FGSG_10999 and FGSG_03624 of the GH11 family, whereas it is ineffective against the two xylanases belonging to family GH10, FGSG_11487 and FGSG_ 11304. These results are in agreement with the notion that TAXI-type XI are typically effective against xylanases of the GH11 family and ineffective against xylanases of family GH10 (Dornez et al. 2010b). In particular, TAXI-I has been tested against the previously mentioned xylanases of $F$. graminearum and the same results have been obtained (Pollet et al. 2009). We also showed a variation in the extent of the inhibition capacity of TAXI-III toward the two inhibited xylanases, FGSG_10999 and FGSG_03624, being more effective against the latter. Worthy of noting is that the genes encoding these two xylanases are the most expressed genes during wheat spike infection by $F$. graminearum (Sella et al. 2013). Consequently, the capacity of TAXI-IIICS to inhibit these two xylanases should play a primary role in counteracting the degradative potential of the pathogen on the cell wall xylan during the infection process. Nonetheless, the inability of TAXI-III to inhibit all four xylanases tested is reflected on its capacity to inhibit only partially the total xylanase activity of $F$. graminearum. Other XI probably could complement the inhibition activity of TAXI-III. Indeed, the two xylanases FGSG_11487 and FGSG_11304, also expressed during $F$. graminearum infection and not inhibited by TAXI-III, are inhibited in vitro by XIP-I (Pollet et al. 2009).

Although TAXI-III is not able to inhibit to completion the total xylanase activity of $F$. graminearum, its overexpression in the transgenic plants correlates with a delayed FHB symptom. In contrast, the inability of TAXI-III to inhibit the xylanase activity secreted by $B$. sorokiniana results in the lack of any reduction of spot blotch symptoms in the TAXI-III plants. These results provide clear evidences of the active contribution of xylanases during the infection of wheat spike tissue by $F$. graminearum and suggest that TAXI-III can control the activity of these enzymes during the infection process. Moreover, although the reduced FHB symptoms in the TAXI-III plants are especially evident in the initial phase of infection, their impact is durable because whole flour from caryopses of TAXIIII plants infected with $F$. graminearum showed a reduced accumulation of fungal biomass.

These results with the TAXI-III plants parallel those previously obtained with transgenic plants expressing a polygalacturonase (PG)-inhibiting protein (PGIP) or a pectin methyl esterase inhibitor (PMEI). Both inhibitors limit the activity of fungal PGs, one of the first CWDE secreted by pathogens during the infection process; and, in both cases, their effectiveness in limiting microbial pathogen colonization of host tissue has been demonstrated in different species. In particular, transgenic wheat expressing PGIP or PMEI showed a reduction of FHB disease symptom development similar to that observed for the TAXI-III plants, with the disease symptoms mostly reduced in the initial-middle stage of infection (Ferrari et al. 2012; Volpi et al. 2011).

In conclusion, we provide, for the first time, clear evidence in planta that XI are involved in plant defense against fungal pathogens. These results and those previously reported with different proteinaceous inhibitors of CWDE, such as PGIP and PMEI, also strengthen the notion that the integrity of host cell wall polysaccharides, regardless of their amount and type, play a key role in keeping the cell wall as a functional barrier against pathogens.

\section{MATERIALS AND METHODS}

\section{Taxi-III isolation and production and characterization of durum wheat transgenic plants.}

Taxi-IIICS, Taxi-IIIBW, and Taxi-IIISV were isolated by PCR from genomic DNA of $T$. aestivum Chinese Spring, $T$. aestivum Bobwhite, and T. durum Svevo, respectively, by using the specific primer pair 101sp(For)/101sp(Rev), developed from the Taxi-IIII sequence of T. aestivum Norin61 (accession number AB114627) (Igawa et al. 2004).

The pUbi::Taxi-IIICS construct was prepared by inserting the complete coding region of Taxi-IIICS into the BamHI site of pAHC17 (Christensen and Quail 1996) under control of the maize Ubiquitinl promoter and NOS terminator. The BamHI sites flanking the complete coding region of Taxi-IIICS were generated by PCR amplification using the forward and reverse primers (Taxi3_BamHI1F) 5'-AATAGGATCCATGGCACGG GTCCTCCTCCTC and (Taxi3_BamHI2R) 5'-AATAGGATCC CTAGCTGCCGCAACCCGTAAAG, respectively. PCR reactions were performed in a reaction volume of $50 \mu \mathrm{l}$ with $10 \mathrm{ng}$ of plasmid DNA (pTaxi-IIICS), $2.5 \mathrm{U}$ of FastStart High Fidelity PCR system (Roche Diagnostics, Monza, Italy), 1× Taq PCR buffer, $50 \mathrm{ng}$ of each of the two primers, and $100 \mu \mathrm{M}$ each deoxyribonucleotide. Amplification conditions were 1 cycle at $95^{\circ} \mathrm{C}$ for $2 \mathrm{~min} ; 30$ cycles at $95^{\circ} \mathrm{C}$ for $30 \mathrm{~s}, 60^{\circ} \mathrm{C}$ for $1 \mathrm{~min}$, and $72^{\circ} \mathrm{C}$ for $1 \mathrm{~min}$; and a final step at $72^{\circ} \mathrm{C}$ for $5 \mathrm{~min}$. After BamHI digestion, the amplicon was ligated into the BamHI site of pAHC17, generating the pUbi::Taxi-IIICS construct $(5,923 \mathrm{bp})$. The correct sequence of pUbi::Taxi-IIICS and the insertion sites were confirmed by nucleic acid sequencing.

For wheat transformation experiments, the plasmid pAHC20 (Christensen and Quail 1996), carrying the bar gene that confers resistance to the bialaphos herbicide, was co-bombarded with pUbi::Taxi-IIICS in a 1:3 molar ratio. Constructs were delivered into immature embryo-derived calli of $T$. durum Svevo by particle bombardment as described by Volpi and associates (2011).

The presence of pUbi::Taxi-IIICS in bialaphos-resistant $\mathrm{T}_{0}$ plants and their progeny was verified by PCR using total DNA obtained from leaf sections of mature plants (Tai and Tanksley 1991). DNA amplification was carried out according to the procedures specified for GoTaqGreen MasterMix (Promega, Milano, Italy) at an annealing temperature of $60^{\circ} \mathrm{C}$ by using the specific primers pair UBI-A2 (5'-CCTGCCTTCATACGCT ATTTATTTGC) and TaxiIII_2R (5'-TGGAAGGGGATGGTG TAGAG) that produces an amplicon of $198 \mathrm{bp}$.

Genomic DNA extraction and Southern blot analysis were performed as reported by Janni and associates (2008). 
Total RNA was extracted from adult plants using the RNasy plant mini kit (Qiagen, Milano, Italy) according to the manufacturer's instructions. qRT-PCR experiments were performed using the iCycler (Bio-Rad Laboratories, Monza, Italy) and the master mix iQTMSYBER Green supermix (Bio-Rad Laboratories) containing the flurogenic SYBER Green I DNA binding dye. Oligonucleotide primer pairs used were Taxi III 170F (5'-GTCCACGTGCGAGGGTAGT) and Taxi III 5R (5'-CG GGTGTTCTCCACTTTGAT) for Taxi-III and Taact77F ( $5^{\prime}$-T CCTGTGTTGCTGACTGAGG) and Taact312R (5'-GGTC CAAACGAAGGATAGCA) for Actin (accession number AB181991). The homolog genes $\mathrm{TaPr}-1-1 / 2 / 3$, located on the short arm of wheat homologous group 5 (Lu et al. 2011), were simultaneously amplified by using previously developed primers for PR1.1 (Lu et al. 2006). The specificity of the primers was verified by nucleotide sequence of the specific amplicon. The Actin gene was used as housekeeping gene. Total reaction volume was $15 \mu \mathrm{l}$ and included $7.5 \mu \mathrm{l}$ of $(2 \times)$ MasterMix, 100 ng of cDNA, $0.5 \mu \mathrm{l}$ of $10 \mu \mathrm{M}$ each forward and reverse primers, and volume adjusted with water. Each reaction was made in triplicate. Reaction conditions were as follows: one cycle at $50^{\circ} \mathrm{C}$ for $2 \mathrm{~min}$ and $95^{\circ} \mathrm{C}$ for $5 \mathrm{~min}$; then, 40 cycles at $95^{\circ} \mathrm{C}$ for $40 \mathrm{~s}, 61^{\circ} \mathrm{C}$ for $40 \mathrm{~s}$, and $72^{\circ} \mathrm{C}$ for $40 \mathrm{~s}$; and, finally, 90 cycles at $55^{\circ} \mathrm{C}$ for $10 \mathrm{~s}$, increasing the set point temperature after cycle 2 by $0.5^{\circ} \mathrm{C}$.

The relative expression analysis was determined by using the 2- $\Delta \Delta \mathrm{CT}$ method (Livak and Schmittgen 2001) (User Bulletin Number 2-P/N 4303859; Applied Biosystems, Foster City, CA, U.S.A.). Calculation and statistical analyses were performed by Gene Expression Macro Version 1.1 (Bio-Rad Laboratories). The qRT-PCR experiments were performed on RNA samples from two different biological replicas.

\section{Fungal growth, heterologous expression, and protein analyses.}

F. graminearum Schwabe strains PH1 and 3824 and Bipolaris sorokiniana (Sacc.) Shoemaker DSMZ 62608 were cultured at $25^{\circ} \mathrm{C}$ on potato dextrose agar (PDA) (Difco Laboratories, Detroit).

Xylanase activity was induced as proposed by Dong and associates (2012), with some modification. Five agar plugs $(5 \mathrm{~mm}$ in diameter), taken from the edge of actively growing colonies, were used to inoculate $250-\mathrm{ml}$ flasks containing $50 \mathrm{ml}$ of synthetic medium supplemented with glucose at $20 \mathrm{~g} /$ liter. These cultures were incubated on an orbital shaker at $25^{\circ} \mathrm{C}$ and 100 rpm. After 3 days of growth, $200 \mu$ of each culture containing the fungal propagules were used to inoculate $4 \mathrm{~g}$ of wheat bran hydrated with $4 \mathrm{ml}$ of modified synthetic medium in petri dishes. The plates were grown for 14 days at $25^{\circ} \mathrm{C}$. Bran-fungal mat $(8 \mathrm{~g})$ was sliced and ground with $40 \mathrm{ml}$ of extraction buffer (50 mM sodium acetate, 10\% glycerol, 2.5 M EDTA, $0.5 \mathrm{M} \mathrm{NaCl}$, and $2.5 \mathrm{M}$ dithiothreitol) containing $0.4 \mathrm{~g}$ of polyvinylpolypyrrolidone. The mixture was filtered through a cheesecloth and the crude extract was collected in a flask, while the solid fraction was used for a second extraction with $20 \mathrm{ml}$ of extraction buffer. The collected filtrates were centrifuged at $8,000 \mathrm{rpm}$ for $15 \mathrm{~min}$ at $4^{\circ} \mathrm{C}$ and supernatants were used to determine the xylanase activity.

The FGSG_03624 endoxylanase gene was cloned and expressed in $P$. pastoris as reported by Sella and associates (2013). The entire coding sequences of FGSG_10999, FGSG_11487, and FGSG_11304 endoxylanase genes were amplified by using the cDNA obtained from total RNA extracted from infected wheat spikelets at 3 dpi. Reverse transcription was performed by using an Oligo(dT) primer and the ImPromII reverse transcriptase (Promega, Milano, Italy), following the manufacturer's instructions. Amplification from cDNA was carried out by using the REDTaq ReadyMix PCR reaction mix (SigmaAldrich, Milano, Italy) with the specific primers pairs reported in Supplementary Table S1. The PCR was performed by repeating for 35 times the following cycle: $1 \mathrm{~min}$ at $94^{\circ} \mathrm{C}, 30 \mathrm{~s}$ at the proper annealing temperature, and 1 to $1.5 \mathrm{~min}$ at $72^{\circ} \mathrm{C}$. The amplification products of the expected size were purified using the "Wizard SV Gel and PCR Clean-Up System" kit (Promega, Milano, Italy) and were then cloned individually into the pGEM-T Easy vector (Promega, Milano, Italy), following the manufacturer's instructions. The cloned cDNAs were sequenced in order to check the correctness of the nucleotide sequences and then amplified with the Pfu DNA polymerase (Promega, Milano, Italy) by using specific primers containing adaptors for $E c o$ RI and $X b a \mathrm{I}$ recognition sequences. The amplification was performed by repeating for 35 times the following cycle: $1 \mathrm{~min}$ at $94^{\circ} \mathrm{C}, 30 \mathrm{~s}$ at the proper annealing temperature, and 1 to 1.5 min at $72^{\circ} \mathrm{C}$. The PCR amplicons were purified and individually ligated into the pPICZ $\alpha \mathrm{A}$ expression vector and each plasmid was used to transform $P$. pastoris by electroporation as reported in Sella and associates (2013).

Some positive colonies were tested by PCR using specific primers and the REDTaq ReadyMix PCR reaction mix (SigmaAldrich). Colonies showing the expected insert were grown and induced with methanol according to the manufacturer's instructions (Invitrogen Life Technologies, Milano, Italy). After 96 h, liquid cultures were centrifuged at $10,000 \times g$ for $10 \mathrm{~min}$ and supernatants were assayed for xylanase activity and subjected to SDS-PAGE. Supernatants from recombinant colonies showed xylanase activity, whereas the supernatant from control colonies (not containing the xylanse genes) did not show any xylanase activity.

SDS-PAGE analysis (12\%) was performed according to Laemmli and associates (1970) by using $25 \mu \mathrm{l}$ of the P. pastoris culture medium. Gels were stained with Coomassie Brilliant Blue R250 (Sigma-Aldrich).

Crude protein extracts were obtained by homogenizing wheat tissues in the presence of McIlvaine's buffer at $\mathrm{pH} 5.0$ (0.2 $\mathrm{M}$ disodium hydrogen phosphate and $0.1 \mathrm{M}$ citric acid). The homogenate was shaken for $1 \mathrm{~h}$ at $4^{\circ} \mathrm{C}$ and centrifuged 20 min at $10.000 \times g$, and the supernatant recovered.

TAXI-III was purified from crude protein extract by affinity chromatography on a xylanase-sepharose conjugate column. Homogeneous $A$. niger endo-1,4- $\beta$-xylanase M4 (Megazyme, Bray, Ireland) was covalently bound to NHS-activated Sepharose 4 Fast Flow (GE Healthcare, Little Chalfont, United Kingdom) according to the manufacturer's instructions. The XylM4-Sepharose conjugate was suspended in McIlvaine buffer ( $\mathrm{pH}$ 5.0) and packed in a column (Poly-Prep Chromatography Column, $9 \mathrm{~cm}$ high, $2-\mathrm{ml}$ bed volume, 0.8 by $4 \mathrm{~cm}$ ) that was used for TAXI-III purification.

$\mathrm{N}$-terminal sequencing and MS analyses of TAXI-III were performed by the Molecular Structure Facility at University of California (Davis). N-terminal amino acid sequencing was performed by means of an Applied Biosystems Procise 494 sequencer. Purified TAXI-III was fractionated on SDS-PAGE and electroblotted with the Mini Trans-Blot Electrophoretic Transfer Cell (Bio-Rad Laboratories), by using $10 \mathrm{mM}$ cleaved amplified polymorphic sequence buffer, $\mathrm{pH} 11$, at $4^{\circ} \mathrm{C}$ for $1 \mathrm{~h}$ at $70 \mathrm{~V}$. Staining was performed with $50 \%$ Methanol and $0.1 \%$ Coomassie blue R-250.

MS analyses were performed on purified TAXI-III previously fractionated by SDS-PAGE, stained with colloidal Coomassie CBBG-250 (Neuhoff et al. 1988), and subjected to tryptic digest procedures according to the In-Gel Digest protocol used by the UC Davis Proteomics Core Facility, where the analyses were performed. Tandem MS (MS/MS) was performed by using Sequest (version SRF v. 3; Thermo Fisher 
Scientific, San Jose, CA, U.S.A.) that was set up to search against the specific TAXI-IIICS protein sequence, assuming the digestion enzyme trypsin. Sequest was searched with a fragment ion mass tolerance of $1 \mathrm{Da}$ and a parent ion tolerance of $2 \mathrm{Da}$. Oxidation of methionine and iodoacetamide derivative of cysteine were specified in Sequest as variable modifications. Scaffold (version Scaffold_3_00_06; Proteome Software Inc., Portland, OR, U.S.A.) was used to validate MS/MS based peptide and protein identifications. Peptide identifications were accepted if they exceeded specific database search engine thresholds. Sequest identifications required at least $\Delta \mathrm{Cn}$ scores of greater than 0.10 and XCorr scores of greater than 1.2, 2.0, 2.5 , and 3.0 for singly, doubly, triply, and quadruply charged peptides, respectively. Protein identifications were accepted if they contained at least two identified peptides.

EFs were extracted from wheat seedlings (Zadoks stage 61) by vacuum-infiltration with McIlvane buffer (discussed above) as described by Salvi and associates (1990). Cytoplasmic contamination was verified by determining the glucose-6-phosphate dehydrogenase activity of the extracts.

Protein concentrations were determined with the Bio-Rad Protein assay kit (Bio-Rad Laboratories).

Xylanase activity of $P$. pastoris cultures expressing $F$. graminearum xylanases (FGSG_11487, FGSG_10999, FGSG_ 11304, and FGSG_03624) was determined by measuring the reducing sugars released from $0.5 \%$ (wt/vol) beech-wood xylan (Carl Roth GmbH, Karlsruhe, Germany) dissolved in 50 $\mathrm{mM}$ sodium citrate buffer at $\mathrm{pH} 5$ according to the DNS method described by Miller (1959) and modified by Bailey and associates (1992). The assay was performed by incubating 50 to $200 \mu \mathrm{l}$ of culture filtrates in a reaction volume of $500 \mu \mathrm{l}$. Absorbance of samples was measured at $545 \mathrm{~nm}$ using monomeric D-xylose as standard. One unit of xylanase activity was defined as the amount of enzyme required to release $1 \mu \mathrm{mol}$ of xylose in 1 min under the assay conditions.

Xylanase inhibition activity of crude plant extract or purified TAXI-III was measured by radial gel diffusion assay or by using the DNS assay. Radial gel diffusion assay was performed using a modification of the procedure reported in Emami and Hack (2000). Total protein extract and $0.005 \mathrm{U}$ of endo-1,4- $\beta$-xylanase M4 of A. niger (AnM4xyl; Megazyme) were added to $0.5-\mathrm{cm}$ wells on plates containing McIlvane buffer (pH 5.0), 1\% (wt/vol) xylan from beech wood (SigmaAldrich), and 1\% agarose. Plates were incubated for $16 \mathrm{~h}$ at $30^{\circ} \mathrm{C}$ and the halo caused by enzyme activity was visualized after 30 min of treatment with ethanol $95 \%$ that revealed clear halo of degradation against an opaque background (Royer and Nakas 1990).

DNS assay was performed with $1 \%$ (wt/vol) low-viscosity wheat arabinoxylan (Megazyme) in a reaction volume of 500 $\mu \mathrm{l}$. Reactions (in triplicate) containing xylanase $(0.015 \mathrm{U}$ of heterologously expressed $F$. graminearum xylanase or $0.050 \mathrm{U}$ of crude filtrate of $F$. graminearum or B. sorokiniana), alone or preincubated $\left(4 \mathrm{~min}\right.$ at $30^{\circ} \mathrm{C}$ ) with protein extract or purified TAXI-III, were assayed for xylanase activity at $40^{\circ} \mathrm{C}$ and terminated after 4 min by adding DNS.

Kinetic parameters were determined using the DNS assay by incubating $0.041 \mathrm{U}$ of AnM4xyl with different concentrations of soluble low-viscosity arabinoxylan $(0.0050$ to $0.0250 \mathrm{mg} / \mathrm{ml})$ in a reaction volume of $250 \mu \mathrm{l} . K_{m}$ and $k_{\text {cat }}$ values were calculated using nonlinear regression Michaelis-Menten equation.

\section{Plant growth, infection assays, and fungal biomass quantification.}

Wheat seeds (T. durum Svevo) were surface sterilized with sodium hypochlorite $(0.5 \%, \mathrm{vol} / \mathrm{vol})$ for $20 \mathrm{~min}$ and then rinsed thoroughly in sterile water. Plants were vernalized at $4^{\circ} \mathrm{C}$ for 2 weeks and grown in a climatic chamber at 18 to $23^{\circ} \mathrm{C}$ with a 14 -h photoperiod $\left(300 \mu \mathrm{E} \mathrm{m}^{-2} \mathrm{~s}^{-1}\right)$.

For plant inoculation with B. sorokiniana 62608 DSMZ, conidia were recovered from fungal cultures grown on petri dishes containing PDA medium (BD, Sparks, MD, U.S.A.). The infection experiment was carried out following the procedure reported by Janni and associates (2008).

Lesion sizes were determined 72 hpi by scanning the leaf surface and calculating the pixels of the infected area and of the total area by using the Adobe Photoshop program (Microsoft, Segrate, Italy). The average area per lesion was calculated as weighted arithmetic mean.

$\mathrm{T}_{1}$ or $\mathrm{T}_{2}$ progenies were used in the different experiments. At least 13 plants for each genotype were used.

For plant inoculation with $F$. graminearum strain 3824, conidia were recovered from fungal cultures grown on synthetic nutrient agar plates (Urban et al. 2002). Infection experiments of wheat plants were performed by single-spikelet inoculation. A conidia suspension $\left(20 \mu \mathrm{l}, 2.5 \times 10^{4}\right.$ conidia $\left.\mathrm{ml}^{-1}\right)$ supplemented with $0.1 \%$ Tween-20 was pipetted directly through the glumes of two opposite central florets of a wheat head during anthesis. Infected spikes were covered with plastic bags for 2 days in order to maintain high humidity. Disease symptoms were assessed by counting the number of visually diseased spikelets at different days postinoculation and by relating them to the total number of spikelets of the respective head, resulting in a percentage of symptomatic spikelets. For each experiments, at least 13 plants of $\mathrm{T}_{2}$ progeny for each genotype were used.

Data from both $B$. sorokiniana or $F$. graminearum infection experiments were analyzed statistically applying the Student's $t$ test.

Quantification of fungal biomass was performed by qPCR assay for the Tri6 gene with the primers Tri6_10F and Tri6_4R (Horevaj et al. 2011). Briefly, at the end of the infection experiments, all dry caryopses of each transgenic and control genotype obtained from each experiment were pooled to make whole wheat flour. Total DNA from whole flour was extracted with DNeasy plant mini kit (Qiagen) according to manufacturer's instructions. qPCR experiments were performed using the iCycler (Bio-Rad Laboratories) and the master mix iQ SYBR Green Supermix (Bio-Rad Laboratories) containing the flurogenic SYBER Green I DNA binding dye. The Actin gene (discussed above) was used as housekeeping gene. The specificity of the primers was verified by nucleotide sequence of the amplicon.

\section{ACKNOWLEDGMENTS}

Research was supported by the Italian Ministry of University and Research (PRIN 2010-2011) and Italian Ministry of Agricultural, Food and Forestry Policies (Grant "Progetto Internazionale di Sequenziamento del Genoma di Frumenti. Mappa fisica del Cromosoma 5A") to R. D'Ovidio. Research was also supported by "Progetto di Ateneo" (2010-prot. CPDA109182) funded by the Padova University to L. Sella.

\section{LITERATURE CITED}

Bailey, M. J., Biely, P., and Poutanen, K. 1992. Interlaboratory testing of methods for assay of xylanase activity. J. Biotechnol. 23:257-270.

Beliën, T., Van Campenhout, S., VanAcker, M., and Volckaert, G. 2005. Cloning and characterization of two endoxylanases from the cereal phytopathogen Fusarium graminearum and their inhibition profile against endoxylanase inhibitors from wheat. Biochem. Biophys. Res. Commun. 327:407-414.

Biely, P., Vrsanská, M., Tenkanen, M., and Kluepfel, D. 1997. Endo-beta1,4-xylanase families: Differences in catalytic properties. J Biotechnol. 57:151-66.

Brito, N., Espino, J. J., and Gonzalez, C. 2006. The endo-beta-1,4-xylanase xyn 11A is required for virulence in Botrytis cinerea. Mol PlantMicrobe Interact. 19:25-32. 
Cantu, D., Vicente, A. R., Labavitch, J. M., Bennett, A. B., and Powell, A. L. T. 2008. Strangers in the matrix: Plant cell walls and pathogen susceptibility. Trends Plant Sci. 13:610-617.

Christensen, A. H., and Quail, P. F. 1996. Ubiquitin promoter-based vectors for high-level expression of selectable and/or screenable marker genes in monocotyledonous plants. Transgenic Res. 5:213-218.

Croes, E., Gebruers, K., Luyten, N., Delcour, J. A., and Courtin, C. M. 2009. The three classes of wheat xylanase-inhibiting proteins accumulate in an analogous way during wheat ear development and germination. J. Plant Physiol. 166:1253-1262.

Debyser, W., Peumans, W. J., Van Damme, E. J. M., and Delcour, J. A. 1999. Triticum aestivum xylanase inhibitor (TAXI), a new class of enzyme inhibitor affecting breadmaking performance. J. Cereal Sci. 30:39-43.

Dong, X., Meinhardt, S. W., and Schwarz, P. B. 2012. Isolation and characterization of two endoxylanase from Fusarium graminearum. J. Agric. Food Chem. 60:2538-2545.

Dornez, E., Croes, E., Gebruers, K., Carpentier, S., Swennen, R., Laukens, K., Witters, E., Urban, M., Delcour, J. A., and Courtin, C. M. 2010a. 2D DIGE reveals changes in wheat xylanase inhibitor protein families due to Fusarium graminearum $\Delta$ Tri5 infection and grain development. Proteomics 10:2303-2319.

Dornez, E., Croes, E., Gebruers, K., De Coninck, B., Cammue, B. P. A., Delcour, J. A., and Courtin, C. M. 2010b. Accumulated evidence substantiates a role for three classes of wheat xylanase inhibitors in plant defense. Crit. Rev. Plant Sci. 29:244-264.

Elliott, G., Durand, A., Hughes, R. K., D’Ovidio, R., and Juge, N. 2009. Isolation and molecular characterisation of a novel xylanase inhibitor gene from durum wheat. J. Cereal Sci. 50:324-331.

Elliott, G. O., Hughes, R. K., Juge, N., Kroon, P. A., and Williamson, G. 2002. Functional identification of the cDNA coding for a wheat endo1,4-beta-D-xylanase inhibitor. FEBS (Fed. Eur. Biochem. Soc.) Lett. 519:66-70.

Emami, K., and Hack, E. 2001. Characterization of xylanase gene from Cochliobolus sativus and its expression. Mycol. Res. 105:352-359.

Ferrari, S., Sella, L., Janni, M., De Lorenzo, G., Favaron, F., and D'Ovidio, R. 2012. Transgenic expression of polygalacturonase-inhibiting proteins in Arabidopsis and wheat increases resistance to the flower pathogen Fusarium graminearum. Plant Biol. 14:31-38.

Fierens, E., Rombouts, S., Gebruers, K., Goesaert, H., Brijs, K., Beaugrand J., Volckaert, G., Van Campenhout, S., Proost, P., Courtin, C. M., and Delcour, J. A. 2007. TLXI, a novel type of xylanase inhibitor from wheat (Triticum aestivum) belonging to the thaumatin family. Biochem. J. 403:583-591.

Gebruers, K., Brijs, K., Courtin, C. M., Fierens, K., Goesaert, H., Rabijns, A., Raedschelders, G., Robben, J., Sansen, S., Sorensen, J. F., Van Campenhout, S., and Delcour, J. A. 2004. Properties of TAXI-type endoxylanase inhibitors. Biochim. Biophys. Acta 1696:213-221.

Horevaj, P., Milus, E. A., and Bluhm, B. H. 2011. A real-time qPCR assay to quantify Fusarium graminearum biomass in wheat kernels. J. Appl. Microbiol. 111:396-406.

Igawa, T., Ochiai-Fukuda, T., Takahashi-Ando, N., Ohsato, S., Takehico, S., Yamaguki, I., and Kimura, M. 2004. New TAXI-type xylanase inhibitor genes are inducible by pathogens and wounding in hexaploid wheat. Plant Cell Physiol. 45:1347-1360.

Igawa, T., Tokai, T., Kudo, T., Yamaguchi, I., and Kimura, M. 2005. A wheat xylanase inhibitor gene, Xip-I, but not Taxi-I, is significantly induced by biotic and abiotic signals that trigger plant defense. Biosci. Biotechnol. Biochem. 69:1058-1063.

Janni, M., Sella, L., Favaron, F., Blechl, A. E., De Lorenzo, G., and D'Ovidio, R. 2008. The expression of a bean PGIP in transgenic wheat confers increased resistance to the fungal pathogen Bipolaris sorokiniana. Mol. Plant-Microbe Interact. 21:171-177.

Laemmli, U. K. 1970. Cleavage of structural proteins during the assembly of the head of bacteriophage T4. Nature 227:680-685.

Livak, K. J., and Schmittgen, T. D. 2001. Analysis of relative gene expression data using Real-Time 17 quantitative PCR and $2-\Delta \Delta C T$ method.
Methods 25:402-408.

Lu, S., Friesen, T. L., and Faris, J. D. 2011. Molecular characterization and genomic mapping of the pathogenesis-related protein 1 (PR-1) gene family in hexaploid wheat (Triticum aestivum L.) Mol. Genet. Genomics 285:485-503.

Lu, Z. X., Gaudet, D., Puchalski, B., Despins, T., Frick, M., and Laroche, A. 2006. Inducers of resistance reduce common bunt infection in wheat seedlings while differentially regulating defence-gene expression. Physiol. Mol. Plant P. 67:138-148.

Lysøe, E., Seong, K., and Kistler, H. C. 2011. The transcriptome of Fusarium graminearum during infection of wheat. Mol. Plant-Microbe Interact. 24:995-1000.

McLauchlan, W. R., Garcia-Conesa, M. T., Williamson, G., Roza, M Ravestein, P., and Maat, J. 1999. A novel class of protein from wheat which inhibits xylanases. Biochem. J. 338:441-446.

Miller, G. L. 1959. Use of dinitrosalicylic acid reagent for determination of reducing sugars. Anal. Chem. 31:426-428

Neuhoff, V., Arold, N., Taube, D., and Ehrhardt, W. 1998. Improved staining of proteins in polyacrylamide gels including isoelectric focusing gels with clear background at nanogram sensitivity using Coomassie Brilliant Blue G-250 and R-250. Electrophoresis 9:255-262.

Paper, J. M., Scott-Craig, J. S., Adhikari, N. D., Cuomo, C. A., and Walton, J. D. 2007. Comparative proteomics of extracellular proteins in vitro and in planta from the pathogenic fungus Fusarium graminearum. Proteomics 7:3171-3183.

Pollet, A., Beliën, T., Fierens, K., Delcour, J. A., and Courtin, C. M. 2009. Fusarium graminearum xylanases show different functional stabilities, substrate specificities and inhibition sensitivities. Enzyme Microb. Technol. 44:189-195.

Raedschelders, G., Fierens, K., Sansen, S., Rombouts, S., Gebruers, K. Robben, J., Rabijns, A., Courtin, C. M., Delcour, J. A., Van Campenhout, S., and Volckaert, G. 2005. Molecular identification of wheat endoxylanase inhibitor TAXI-II and the determinants of its inhibition specificity. Biochem. Biophys. Res. Commun. 335:512-522.

Royer, J. C., and Nakas, J. P. 1990. Interrelationship of xylanase induction and cellulase induction of Trichoderma longibrachiatum. Appl. Environ. Microbiol. 56:2535-2539.

Salvi, G., Giarrizzo, F., De Lorenzo, G., and Cervone, F. 1990. A Polygalacturnase-Inhibiting Protein in the flowers of Phaseolus vulgaris. L. J. Plant Physiol. 136:513-518.

Sella, L., Gazzetti, K., Faoro, F., Odorizzi, S., D’Ovidio, R., Schäfer, W., and Favaron, F. 2013. A Fusarium graminearum xylanase expressed during wheat infection is a necrotizing factor but is not essential for virulence. Plant Physiol. Biochem. 64:1-10.

Tai, T. H., and Tanksley, S. D. 1991. A rapid and inexpensive method for isolation of total DNA from dehydrated plant tissue. Plant Mol. Biol. Rep. 8:297-303.

Takahashi-Ando, N., Inaba, M., Ohsato, S., Igawa, T., Usami, R., and Kimura, M. 2007. Identification of multiple highly similar XIP-type xylanase inhibitor genes in hexaploid wheat. Biochem. Biophys. Res. Commun. 360:880-884.

Urban, U., Daniels, S., Mott, E., and Hammond-Kosack, K. 2002. Arabidopsis is susceptible to the cereal ear blight fungal pathogens Fusarium graminearum and Fusarium culmorum. Plant J. 32:961-973.

Volpi, C., Janni, M., Lionetti, V., Bellincampi, D., Favaron, F., and D'Ovidio, R. 2011. The ectopic expression of a pectin methyl esterase inhibitor increases pectin methyl esterification and limits fungal diseases in wheat. Mol. Plant-Microbe Interact. 24:1012-1019.

\section{AUTHOR-RECOMMENDED INTERNET RESOURCES}

The Carbohydrate-Active enZYmes database: www.cazy.org

The SignalP 4.1 server: www.cbs.dtu.dk/services/SignalP

University of California Davis Proteomics Core Facility: http://proteomics.ucdavis.edu 